\title{
Differential levels of human leukocyte antigen-class I, multidrug- resistance 1 and androgen receptor expressions in untreated prostate cancer cells: The robustness of prostate cancer
}

\author{
SHIGENORI HOMMA ${ }^{1,3}$, YOSHIHIRO KOMOHARA ${ }^{1}$, MAMORU HARADA $^{1}$, HIDEYUKI SAYA $^{4}$, \\ SATORU TODO $^{3}, \mathrm{KYOGO} \mathrm{ITOH}^{1}$ and MASANORI NOGUCHI ${ }^{2}$ \\ Departments of ${ }^{1}$ Immunology and ${ }^{2}$ Urology, Kurume University School of Medicine, 67 Asahi-machi, Kurume, \\ Fukuoka 830-0011; ${ }^{3}$ Department of General Surgery, Hokkaido University Graduate School of Medicine, \\ N15 W7, Sapporo, Hokkaido 060-8788; ${ }^{4}$ Department of Oncology, Kumamoto University \\ Graduate School of Medicine, 1-1-1 Honjo, Kumamoto, Japan
}

Received March 6, 2007; Accepted April 23, 2007

\begin{abstract}
Tumors are highly robust and maintain their proliferative potential against a wide range of both hostdefense mechanisms and anticancer therapies. One of the approaches to overcome cancer robustness could be combined therapy in which each modality imposes independent selective pressures against the acquired mutation of cancer. To develop such a therapy, it is crucial to understand the magnitude of acquired mutations. In this study, we investigated the levels of human leukocyte antigen (HLA)-class I, multidrugresistance 1 (MDR1), and androgen receptor (AR) expressions in untreated prostate cancers harvested by radical prostatectomy. The mean percentages of cancer cells expressing HLA-class I, MDR and AR among the 10 cancer samples were 41,35 and $74 \%$, respectively. In addition, double-staining of HLA and MDR revealed the four definite populations $\left(\mathrm{HLA}^{+} / \mathrm{MDR}^{+}, \mathrm{HLA}^{+} / \mathrm{MDR}^{-}, \mathrm{HLA}^{-} / \mathrm{MDR}^{+}\right.$and $\left.\mathrm{HLA}^{-} / \mathrm{MDR}^{-}\right)$ in cancer tissues from the majority of cancer patients tested, and the mean percentages of cells expressing these combinations were 13, 29, 22 and 38\%, respectively. Similar results were obtained by double-staining of HLA and AR, except for 2 cases in which $\mathrm{HLA}^{-} / \mathrm{AR}^{+}$cancer cells predominated. These results indicated that untreated prostate cancer cells acquired a wide range of genomic mutations, which may have been caused by internal host pressure to eliminate malignant cells, and would provide evidence of the robustness of untreated prostate cancer.
\end{abstract}

Correspondence to: Dr Masanori Noguchi, Department of Urology, Kurume University School of Medicine, 67 Asahi-machi, Kurume, Fukuoka 830-0011, Japan

E-mail: noguchi@med.kurume-u.ac.jp

Key words: prostate cancer, HLA-class I, MDR1, androgen receptor, mutation

\section{Introduction}

It is generally accepted that cancer is an extremely complex and heterogeneous disease that exhibits a high level of robustness against a range of both host-defense systems and therapeutic efforts (1-3). Robustness allows tumors to survive and grow in several ways. Heterogeneity among tumor cells provides a high level of redundancy, and hence increased chances of survival and growth, which are further enhanced by feedback controls at the cellular level. One of the approaches to overcome cancer robustness could be to understand the magnitude of mutations and then to design a new combination therapy in which each modality imposes independent selective pressures against the acquired mutation of cancer.

Loss of human leukocyte antigen (HLA)-class I expression is considered to be a major mechanism of tumor cell escape from immune surveillance (4-6), while appearance of multidrug-resistance 1 (MDR1) is the major mechanism of tumor cell resistance to chemotherapy $(7,8)$. Mutation and amplification of the androgen receptor (AR) gene are also considered to be responsible for androgen-independence of prostate cancer cells $(9,10)$. Thus all three genetic mutations play a role in the robustness of prostate cancers.

To better understand the magnitude of these mutations, we investigated the levels of HLA-class I, MDR1 and AR expressions in untreated prostate cancers removed by radical prostatectomy, and found that untreated prostate cancer cells had already acquired a wide range of genomic mutations.

\section{Materials and methods}

Patients and tissue samples. Prostate carcinomas were obtained from 10 prostate cancer patients (age range 57-72, median 66.5 years) who had undergone radical prostatectomy for clinically localized T1-2N0M0 prostate cancer between 2005 and 2006 at the Kurume University Hospital. None of the patients had received preoperative therapy, such as androgen deprivation therapy. All patients had an elevated serum level of PSA. Gleason scores ranged from 6 to 9 
(median 7), and most of the carcinomas were well-tomoderately differentiated.

Each tissue sample was divided into the two pieces. One was processed routinely and used for histological finding. The other one was immediately covered with Tissue-Tek (Sakura Finetek USA, Inc., Torrance, CA) frozen tissue embedding medium and stored in liquid nitrogen until use. Frozen sections were obtained from stored tissue, alcohol-fixed, stained with $\mathrm{H} \& \mathrm{E}$, and viewed under a light microscope to confirm the tissue origin and diagnosis.

Immunohistochemical analysis. The prostate specimens were cut into $4-\mu \mathrm{m}$ thick sections using a cryostat, then mounted on silane-coated slides and fixed in cold acetone. After washing in phosphate-buffered saline (PBS), the sections were incubated with $5 \%$ FCS for $20 \mathrm{~min}$, reacted with mouse anti-human HLA-class I monoclonal antibody (Ancell Corp., Bayport, $\mathrm{MN}$ ) and rabbit anti-human MDR/P-glycoprotein polyclonal antibody (Santa Cruz Biotechnology, Santa Cruz, CA) or rabbit anti-human androgen receptor (AR) polyclonal antibody (Santa Cruz Biotechnology) overnight at $4^{\circ} \mathrm{C}$, then washed again in PBS. Control slides were incubated with normal mouse, rabbit immunoglobulins (Santa Cruz). Excess antibody was washed out with PBS, the samples were incubated with HRP-labeled goat anti-mouse or rabbit antibody (Nichirei, Tokyo, Japan) for $60 \mathrm{~min}$. The reaction was visualized by use of the diaminobenzidine substrate system (Dako, Carpinteria, CA). Slides were counterstained with hematoxylin.

For immunofluorescent double-staining, Alexa Fluor 488 goat anti-rabbit IgG (Invitrogen, Groningen, The Netherlands) and Texas Red chicken anti-mouse IgG (Santa Cruz Biotechnology) were used as the secondary antibodies. Tissue sections were read independently by the two investigators. For each sample, the percentage of cells staining positive for each of the three mutations was determined.

\section{Results}

We first examined the expression of HLA-class I antigen in prostate cancer specimens, and the mean percentage of HLAclass I-positive cancer cells among the 10 patients was $41 \%$, with a range of $5-90 \%$. In contrast, this antigen was expressed in all the non-cancerous prostate cells tested. Representative results are given in Fig. 1A. We next investigated the expression of MDR1 in prostate cancer specimens, and the mean percentage of cancer cells expressing MDR1 was $35 \%$, with a range of 2-80\%. In contrast, MDR1 was expressed in only $5-10 \%$ of non-cancerous epithelial cells, which was in agreement with the result of a previous report (8).

Representative results are given in Fig. 1B. We also investigated the expression of $\mathrm{AR}$, and the mean percentage of AR-positive cancer cells was 74\%, with a range of $45-100 \%$. AR was expressed in all the tumor cells from only 3 of the 10 patients (patients 7,9 and 10). In contrast, it was expressed in all the non-cancerous prostate cells tested. Representative results are given in Fig. 1C. It is worth noting that each of these expression levels of HLA-class I antigen, MDR1 and AR in the 10 specimens had no correlation with preoperative PSA levels, clinical stages or pathological risk factors, including organ-confined status, positive surgical margin and seminal vesicle invasion.

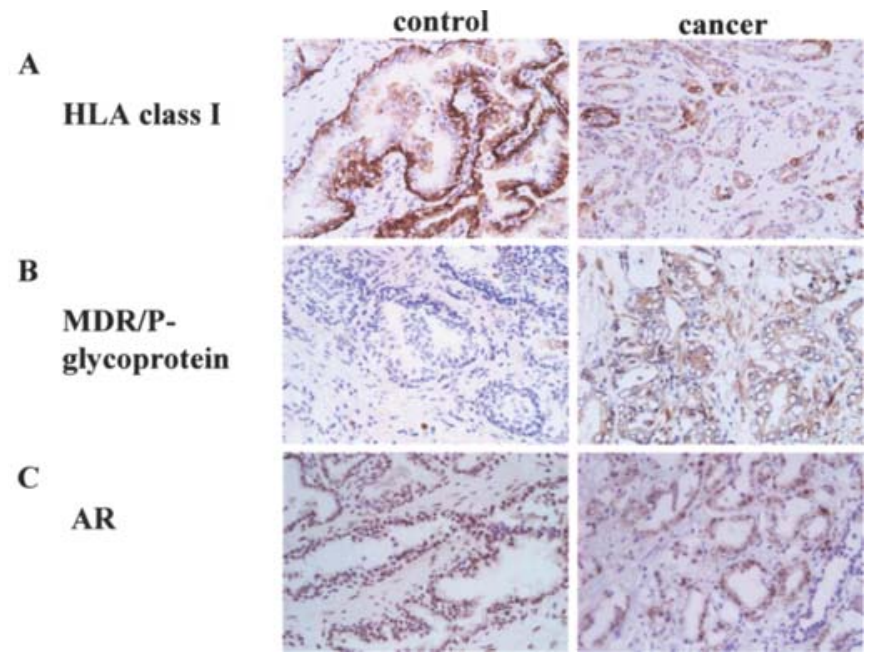

Figure 1. Immunohistochemical staining with anti-HLA-class I (A), MDR1/ P-glycoprotein (B) and androgen receptor (AR) (C) antibody. The expression of the HLA-class I, MDR1 and AR protein in prostate cancer tissues was evaluated by immunohistochemical staining on frozen tissues sections. Control samples were from benign prostate hyperplasia tissues. Representative photographs of prostate cancer are shown. The magnification was x200 in all cases.

Double-staining for HLA-class I and MDR1 expression was also carried out. Representative results are given in Fig. 2A and $\mathrm{B}$, and a summary of the data is shown in Fig. 2C. The percentage of cancer cells co-expressing both HLA-class I and MDR1 antigens ranged from 0 to $50 \%$ among the 10 specimens, with a mean of $13 \%$, whereas those expressing neither of the two ranged from 5 to $95 \%$ with a mean of $38 \%$. The percentage of cancer cells expressing HLA-class I but not MDR1 also ranged from 3 to $65 \%$ among the 10 specimens, with a mean of $29 \%$, while those expressing MDR1 but not HLA-class I ranged from 0 to $65 \%$ with a mean of $22 \%$. There were no significant levels of correlation between HLA-class I antigen and MDR1 expression (Fig. 2D). In contrast, the vast majority of the non-cancerous prostate cells expressed HLAclass I antigen, but not MDR1 (data not shown).

Double-staining for HLA-class I and AR expression was also carried out. Representative results are given in Fig. 3A and $\mathrm{B}$, and the data are summarized in Fig. 3C. The cancer cells co-expressing both HLA-class I and AR ranged from 2 to $80 \%$ among the 10 specimens, with a mean of $31 \%$, whereas those expressing neither of the two ranged from 0 to $63 \%$ with a mean of $18 \%$. The cancer cells expressing HLA-class I but not AR ranged from 0 to $35 \%$ with a mean of $10 \%$, while those expressing AR but not HLA-class I ranged from 10 to $95 \%$ with a mean of $41 \%$. $\mathrm{HLA}^{-} / \mathrm{AR}^{+}$cancer cells predominated in 2 of the 10 patients (patients 7 and 9). There was no correlation between HLA-class I and AR expression (Fig. 3D). In contrast, all the non-cancerous prostate cells expressed both HLA-class I and AR antigens (data not shown).

\section{Discussion}

Loss of HLA-class I expression on the surface of cancer cells is a major mechanism by which tumor cells escape from the host immune system $(5,6)$. It has been well reported in untreated prostate cancer cells. Therefore, the results shown 
A

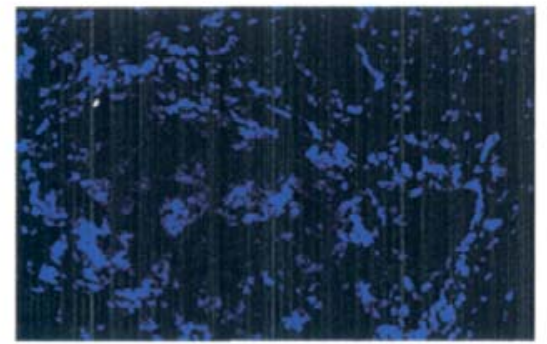

DAPI: blue

C

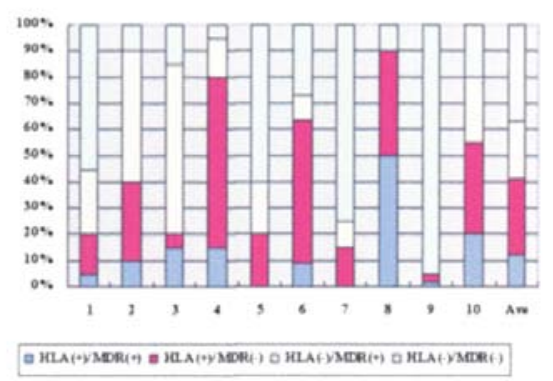

B

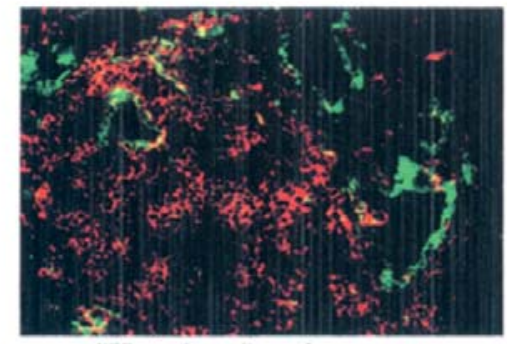

HLA class I: red

MDR/P-glycoprotein: green

D

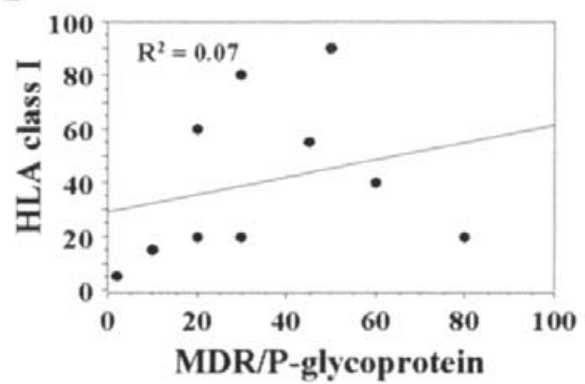

Figure 2. Differential levels of HLA-class I, MDR and AR in case 1 with untreated prostate cancer. (A) DAPI was used for nuclear staining. (B) Immunofluorescent double-staining with HLA-class I (red) and MDR (green). Heterogeneous expression of HLA-class I and MDR was observed in cancer cells. (C) Four populations of $\mathrm{HLA}^{+} / \mathrm{MDR}^{+}, \mathrm{HLA}^{+} / \mathrm{MDR}^{-}, \mathrm{HLA}^{-} / \mathrm{MDR}^{+}$and $\mathrm{HLA}^{-} / \mathrm{MDR}^{-}$in cancer tissues. (D) The correlation between HLA-class I and MDR expression.

A

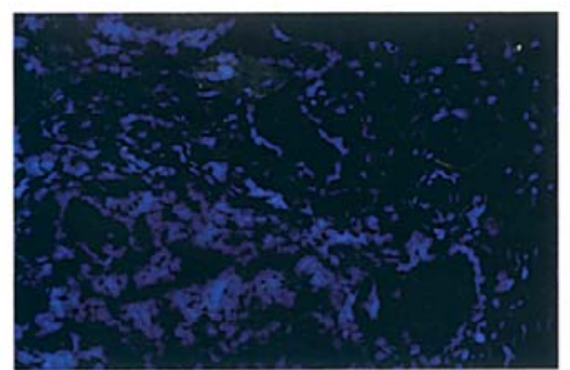

C

DAPI: blue

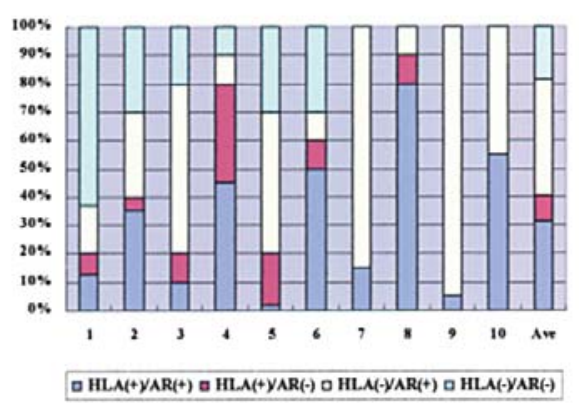

B

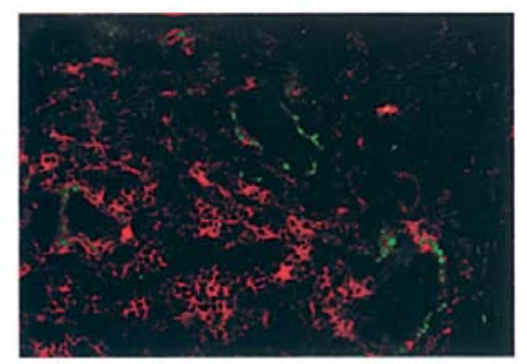

HLA class I: red

Androgen receptor: green

D

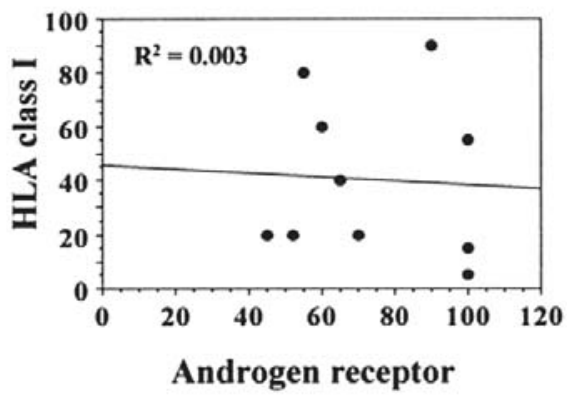

Figure 3. Differential levels of HLA-class I, MDR and AR in case 2 with untreated prostate cancer. (A) DAPI was used for nuclear staining. (B) Immunofluorescent double-staining with HLA-class I (red) and AR (green). Heterogeneous expression of HLA-class I and AR was observed in cancer tissues. (C) Four populations of $\mathrm{HLA}^{+} / \mathrm{AR}^{+}, \mathrm{HLA}^{+} / \mathrm{AR}^{-}, \mathrm{HLA}^{-} / \mathrm{AR}^{+}$and $\mathrm{HLA}^{-} / \mathrm{AR}^{-}$in cancer tissues. (D) The correlation between $\mathrm{HLA}^{-c l a s s ~ I ~ a n d ~} \mathrm{AR}$ expression.

in this study are consistent with those previous reports. Multidrug resistance is one of the most serious causes of failure of chemotherapy, and the magnitude of resistance usually depends on the expression of MDR1 induced by chemotherapy $(7,8)$. We report herein, however, that $35 \%$ of untreated prostate cancer cells showed MDR1 expression, suggesting that a certain innate mechanism other than the immune system is involved in the elimination of prostate cancer cells, which in turn provides selective pressure for prostate cancer cells to acquire MDR1 expression. Androgen blockade has become a 
standard treatment modality for untreated prostate cancers, since it is assumed that a vast majority of these cancers express AR. In the present study, however, we found that the mean percentage of AR-positive untreated cancer cells was $75 \%$ among the 10 patients, with a range of $45-100 \%$. AR was expressed in all the tumor cells from only 3 of the 10 patients (patients 7,9 and 10). These results suggest that innate hormone system provides strong select pressure to prostate cancer cells.

These results, from a single-staining technique, indicated that untreated prostate cancers are highly robust in their growth and survival against various internal perturbations, as pointed out by Kitano (1-3). Therefore, we investigated the heterogeneity of mutations in prostate cancer cells by means of a double-staining technique, and reported that the four definite populations $\left(\mathrm{HLA}^{+} / \mathrm{MDR}^{+}, \mathrm{HLA}^{+} / \mathrm{MDR}^{-}, \mathrm{HLA}^{-} / \mathrm{MDR}^{+}\right.$ and $\left.\mathrm{HLA}^{-} / \mathrm{MDR}^{-}\right)$were present in cancer tissues from the majority (7 of 10) of cancer patients tested. There were no significant levels of correlation between HLA-class I antigen loss and MDR1 expression, indicating that these events are independent of each other. It is worth noting, however, that the mean percentage of cancer cells expressing MDR1 but not HLA-class I, which are expected to be resistant to both chemotherapy and specific immunotherapy, was $22 \%$ among the 10 samples. Therefore, chemotherapy combined with immunotherapy might be more effective than either chemotherapy or immunotherapy alone for untreated prostate cancer.

The four definite populations $\left(\mathrm{HLA}^{+} / \mathrm{AR}^{+}, \mathrm{HLA}^{+} / \mathrm{AR}^{-}\right.$, $\mathrm{HLA}^{-} / \mathrm{AR}^{+}$and $\left.\mathrm{HLA}^{-} / \mathrm{AR}^{-}\right)$were also present in cancer tissues from the majority ( 6 of 10 ) of the cancer patients tested. Therefore, for these patients, hormone therapy combined with immunotherapy might be more effective than hormone therapy or immunotherapy alone.

We found that the mean percentages of cancer cells expressing HLA-class I, MDR, and AR among the 10 samples were 41,35 and $74 \%$, respectively. These results suggest that a hormone therapy alone is more effective than either immunotherapy or chemotherapy. Indeed, maximum androgen block is the standard therapy for untreated prostate cancer, whereas neither immunotherapy nor chemotherapy is used as the first line therapy. However, the majority of patients become resistant to maximum androgen block, which might in part be expected from the results of this study showing that untreated prostate cancer cells had already acquired a wide range of genomic mutations. Collectively, these results provide evidence of the robustness of untreated prostate cancers, and might provide clues for overcoming that robustness.

\section{Acknowledgements}

This study was supported in part by a Grant-in-Aid from the Ministry of Education, Science, Sports and Culture of Japan (No. 12213134 to K.I.) and by a grant from the Research Center for Innovative Cancer Therapy of the 21st Century COE Program for Medical Science (to M.H., M.N and K.I.).

\section{References}

1. Kitano H: Computational systems biology. Nature 420: 206210, 2002.

2. Kitano H: Cancer robustness: tumor tactics. Nature 426: 125 , 2003.

3. Kitano H: Cancer as a robust system: implications for anticancer therapy. Nat Rev 4: 227-235, 2004.

4. Blades RA, Keating PJ, McWilliam LJ, et al: Loss of HLA class I expression in prostate cancer: Implications of immunotherapy. Urology 46: 681-687, 1995.

5. Hicklin DJ, Marincola FM and Ferrone S: HLA class I antigen down-regulation in human cancer: T-cell immunotherapy revives as old story. Mol Med Today 5: 178-186, 1999.

6. Marincola FM, Jaffee EM, Hicklin DJ and Ferrone S: Escape of human solid tumors from T-cell recognition: molecular mechanisms and functional significance. Adv Immunol 74: 181-273, 2000.

7. Persidis A: Cancer multidrug resistance. Nat Biotechnol 17: 94-95, 1999.

8. Kawai K, Sakurai M, Sakai T, et al: Demonstration of MDR1 $\mathrm{P}$-glycoprotein isoform expression in benign and malignant human prostate cells by isoform-specific monoclonal antibodies. Cancer Lett 150: 147-153, 2000.

9. Taplin ME, Bubley GJ, Shuster TD, et al: Mutation of the androgen-receptor gene in metastatic androgen-independent prostate cancer. New Eng J Med 332: 1393-1398, 1995.

10. Tilley WD, Bunchanan G, Hickey TE and Bentel JM: Mutations in the androgen receptor gene are associated with progression of human prostate cancer to androgen independence. Clin Cancer Res 2: 277-285, 1996. 